\title{
Indicadores para la medición de los costos ambientales en entidades de alojamiento turístico: Una necesidad ante la sostenibilidad del uso de los recursos.
}

\author{
Indicators for the measurement of environmental costs in tourist \\ accommodation entities: A need facing the sustainability of the use of \\ resources.
}

MSc. Lidie Perera Conde. ${ }^{1}$, Anel Nogueiras Valdés. ${ }^{2} \&$ Rafael Reinier Alcober Álvarez ${ }^{3}$

\begin{abstract}
The relevance of the use of environmental management accounting in decision making is aimed at the sustainability of resources, however, Cuban companies do not consider in their accounting the environmental costs they generate, since the associated accounting culture is still limited to the environment and the normative base in this sense is scarce. Greater importance is gained by tourism companies, which demand a large consumption of resources, impact the environment and in turn depend on them as tourist attractions. The main objective of this research was to propose indicators to measure the environmental costs derived from the business management of the Roc Presidente Hotel. The research techniques used were documentary and content analysis, as well as the interview, and the data was collected from primary and secondary information sources. The results obtained led to identify and describe the fundamental aspects of the hotel's environmental management and propose indicators related to it, which will allow the entity to measure annual environmental costs and make decisions aimed at achieving more efficient Environmental Management.
\end{abstract}

Key Words: Environmental Indicators, Environmental Costs, Environmental Accounting, Tourism Entities, Hotel.

\section{Resumen}

\footnotetext{
${ }^{1}$ Facultad de Turismo. Universidad de La Habana, Cuba. lidie_perera@ftur.uh.cu

${ }^{2}$ Lic. Anel Nogueira, CUBATUR Ventas Internacionales. Dpto. Eventos e Incentivos, La Habana, Cuba. incentivos.anel.nogueira@ central.cbt.tur.cu

${ }^{3}$ Rafael Reinier Alcober Álvarez. Estudiante 4to año. Facultad de Turismo. Universidad de La Habana. Cuba.rafael.alvarez@estudiantes.ftur.uh.cu
} 
La pertinencia del uso de la contabilidad de gestión ambiental en la toma de decisiones está dirigida a la sostenibilidad de los recursos, sin embargo, las empresas cubanas no consideran en su contabilidad los costos ambientales que generan, ya que aún es limitada la cultura contable asociada al medioambiente y es escasa la base normativa en este sentido. Mayor relevancia ganan las empresas turísticas, que demandan gran consumo de recursos, impactan el medio y a su vez dependen de ellos como atractivos turísticos. La presente investigación tuvo como objetivo general proponer indicadores que permitan medir los costos ambientales derivados de la gestión empresarial del Hotel Roc Presidente. Se aplicaron las técnicas investigativas de análisis documental y de contenido, además de la entrevista, y los datos fueron recolectados de fuentes de información primaria y secundaria. Los resultados obtenidos conllevaron a identificar y describir los aspectos fundamentales de la gestión ambiental del hotel y proponer indicadores afines a dicha gestión que le permitirán a la entidad medir los costos ambientales anuales y tomar decisiones encaminadas a lograr una Gestión Ambiental más eficiente.

Palabras Clave: Indicadores Ambientales, Costos Ambientales, Contabilidad Ambiental, Entidades Turísticas, Hotel, Sostenibilidad.

\section{Introducción}

En los últimos años el cúmulo de impactos medioambientales negativos ocasionados por la humanidad en el planeta ha alcanzado la gravedad suficiente como para que la sociedad no solo haya tomado conciencia sobre este problema, sino que además exija la adopción de soluciones concretas que garanticen la supervivencia en la Tierra de las generaciones del presente y las del futuro.

La culpa del actual deterioro ambiental ha recaído generalmente sobre las actividades industriales y el transporte, sin embargo, otros sectores como el turismo también han provocado impactos al entorno natural y sociocultural de las regiones donde se desarrollan. El producto turístico incorpora como parte inseparable y esencial de sí mismo una serie de recursos naturales (Martín, 2010) como el clima, la vegetación, las playas, montañas, paisajes, y la fauna. Por lo tanto, el turismo no puede prosperar sin una adecuada calidad del medio ambiente, de ahí la necesidad de desarrollar un turismo sostenible para garantizar que este sector económico tan imprescindible para muchos países, incluido Cuba, se ejecute de forma duradera y armónica con el entorno natural. Solo así se podrá mejorar y preservar el patrimonio natural y cultural de las regiones, optimar la calidad de vida de la población residente, y lograr una mayor satisfacción de la demanda turística cada vez más consciente de la problemática ambiental que afronta el mundo en la actualidad.

El desarrollo y la instrumentación de políticas y estrategias nacionales de turismo sustentable constituyen actualmente un tema de interés global que se ha venido expresando en la comunidad internacional a través de recomendaciones de varios organismos. Junto con el resto de la humanidad, Cuba también aboga por el cuidado del medio ambiente como expresa el Artículo 27 de la Constitución de la República y la Ley No.81 de Medio Ambiente. Dentro de estas políticas también se incluye el accionar del 
sector turístico en la isla, que encuentra un espacio en los Lineamientos Económicos del Partido Comunista de Cuba haciendo énfasis en el desarrollo de un turismo sostenible.

Esta preocupación del gobierno y la sociedad por el deterioro del medio ambiente provoca una presión creciente sobre las empresas para que éstas asuman un comportamiento más respetuoso con el entorno en que se desempeñan, animando a los gestores empresariales a asumir la responsabilidad medioambiental que les corresponde; al tiempo que les permite afrontar lo que para sus empresas constituye un reto y, a la vez, una oportunidad competitiva, a través de un desempeño ambiental aceptado por la colectividad.

El sector turístico y sus respectivas empresas, entre ellas los establecimientos de alojamiento turístico, no escapan de esta situación. Los servicios prestados por los hoteles poseen implicaciones ambientales negativas, en ellos se consume agua, energía, se generan volúmenes de residuales de todo tipo y se ocupa un espacio geográfico donde una mala gestión contribuye a su degradación, en ocasiones de forma irreversible. Por este motivo los hoteles se están viendo en la necesidad de llevar a cabo prácticas amigables con el entorno natural y sociocultural con el que interactúan, teniendo en cuenta que el medio ambiente aparece como un factor clave en la selección de la oferta turística en una sociedad cada vez más concienciada sobre los problemas medioambientales y los efectos que sobre ese entorno pueden tener los productos de los que disfruta en sus viajes o momentos de ocio. Teniendo en cuenta este rol a asumir por las empresas turísticas, se hace necesario que las mismas cuenten con herramientas científicas que visualicen y cuantifiquen los beneficios y el deterioro que puede proporcionar o sufrir el medio ambiente como parte inseparable de la misma, así como el costo de las acciones que decidan para su cuidado, y mostrar en sus informes económicos - financieros la información resultante de su relación con el medio ambiente (Paula, 2011).

Actualmente la práctica contable sigue teniendo un dominio económico, pero la presión social y académico-investigativa advierten una ruptura del quehacer tradicional contable, para transitar, de los informes económicos, hacia los informes socio-ambientales de la sostenibilidad. La contabilidad ambiental pretende ampliar el campo de la contabilidad general, incorporando los efectos medioambientales de las actividades económicas de las empresas; partiendo de la contabilización de los costos asociados a situaciones en las que las mismas deben emplear recursos para la gestión y cuidado del medio ambiente (Paula, 2011). El empleo de esta ciencia de la información económica facilita las decisiones directivas relativas a la actuación ambiental de la empresa a partir de la selección de indicadores, la recogida y análisis de datos, la evaluación de esta información con relación a los criterios de actuación ambiental, la comunicación, la revisión y mejora periódicos de tales procedimientos.

Estudios preliminares realizados sobre este particular, de pregrado Paula (2011), Feria (2012), Pérez (2013), Suárez (2013) y de posgrado Vargas (2013), resaltan la importancia del desarrollo sostenible del turismo, la necesidad de lograr una gestión ambiental 
eficiente y sostenible; así como la identificación y cuantificación de los costos ambientales derivados de los impactos, los riesgos y la vulnerabilidad de las empresas turísticas, en especial las dedicadas al alojamiento en los destinos turísticos. Según la revisión bibliográfica realizada se ha validado la necesidad de que las mismas cuenten con herramientas como la contabilidad ambiental que permitan cuantificar la relación turismo - medio ambiente en la gestión de los destinos turísticos.

La actualidad y pertinencia de la temática que se aborda demuestra que el desarrollo de la contabilidad de gestión ambiental es una necesidad en la toma de decisiones dirigida a la sostenibilidad de los recursos naturales, sin embargo, las empresas cubanas no tienen en cuenta en su contabilidad los costos ambientales que generan, ya que aún es limitada la cultura contable asociada al medioambiente y no existe una base normativa en este sentido. De esta situación no están exentas las empresas turísticas cubanas, entre ellas el Hotel Roc presidente, localizado en Calzada No. 110 esquina G, Vedado, Municipio Plaza de la Revolución; Provincia La Habana. Los directivos y trabajadores del hotel tienen como principio el cuidado del medio ambiente y el perfeccionamiento de la gestión ambiental y resaltan de gran importancia la necesidad de contar con alguna herramienta que les permita identificar los costos ambientales derivados de su gestión, en aras de hacer más efectiva la toma de decisiones. Para ello se definió como objetivo general: proponer indicadores que permitan medir los costos ambientales derivados de la gestión empresarial del Hotel Roc presidente.

\section{Metodología}

La presente investigación es de tipo descriptiva ya que el propósito fue referir las principales características de la actuación ambiental del Hotel Roc presidente, así como de la contabilidad del hotel, analizando algunos procedimientos que tributan al cálculo de los costos ambientales y otros indicadores. Además, tiene carácter no experimental porque se realizó sin manipular deliberadamente las variables; se observaron fenómenos ya existentes tal y como se dieron en su contexto actual, y después fueron analizados. Es de tipo transversal ya que se recolectaron los datos en un sólo momento, en un tiempo único, su propósito fue describir variables y analizar su incidencia e interrelación en un momento dado, presentándose un panorama del estado de las variables exploradas en la investigación en determinado momento (Hernández, 2006).

Según Perelló (2005), las fuentes de información pueden clasificarse en primarias y secundarias, y ambas; según su procedencia pueden ser externas o internas, es decir, del entorno empresarial o de la propia empresa. La información primaria es la que se capta expresamente para la investigación emprendida, mientras que la secundaria es aquella que ya está disponible en el momento de comenzar esta. La información secundaria interna se obtuvo en la revisión de documentos del Hotel Roc Presidente como el organigrama de la institución, los objetivos para el año 2018, los indicadores económicos, se analizaron el Plan anual de Capacitación y Desarrollo del 2017, los registros del consumo de agua, electricidad, gas y combustible, el Balance General y el Estado de 
Resultados al cierre del 2017, los registros de la recopilación de materia prima y el Informe sobre el cumplimiento de los puntos de la Guía para la Inspección Ambiental Estatal a Hoteles. Por otra parte, la fuente de información secundaria externa provino fundamentalmente de libros, revistas, monografías, informes de investigaciones en opción a diferentes grados del nivel superior (trabajos de diploma), sitios de internet, normas nacionales e internacionales, y la reglamentación ambiental vigente en el país. También fueron tomados como fuente de información algunos proyectos de investigación de organizaciones extranjeras como la Asociación Española de Contabilidad y Administración de Empresas, División de Naciones Unidas para el Desarrollo Sostenible, Agencia Europea de Medio Ambiente, el Ministerio Federal de Medio Ambiente de Alemania, y otras.

Para la elaboración de esta investigación se emplearon varias técnicas, tales como: el análisis documental, análisis de contenido y la entrevista.

Análisis documental: se empleó como herramienta clave en la definición de los conceptos bases de la temática que se aborda y para conocer las referencias actuales a ella. Fue de gran utilidad para la creación del marco teórico y referencial sobre las particularidades de la contabilidad en el sector hotelero, así como las características fundamentales de los estados financieros. También permitió caracterizar la entidad objeto de estudio. Además, los documentos revisados permitieron un mayor conocimiento y objetividad sobre el tema tratado.

Análisis de contenido: se aplicó en el análisis de los estados financieros del hotel y de los indicadores económicos, cuyo propósito básico fue diagnosticar las posibilidades que brindan para la determinación de los indicadores de costos ambientales.

La entrevista: se empleó como técnica cualitativa de recogida de información primaria, con la intención de obtener información brindada por algunos especialistas del hotel sobre la actuación ambiental y la integración de aspectos ambientales en el sistema contable.

Para el cumplimiento de cada una de las etapas se emplearon métodos de investigación teóricos como: Histórico-Lógico (para la consulta de la bibliografía disponible relacionada con el tema, el cual permitió el estudio y planteamiento cronológico de las citas de autores en el marco conceptual y en la caracterización de la estrategia ambiental seguida por el objeto de estudio); Análisis-Síntesis (en el procesamiento de la información obtenida para caracterizar la situación ambiental actual que presenta el Hotel Roc Presidente, además en el análisis de los estados financieros de la entidad y de los datos aportados por los cuestionarios); Inductivo - Deductivo (para arribar a conclusiones generales válidas).

El método empírico utilizado fue la observación no participativa. El uso de este método permitió tener una percepción objetiva de la realidad del Hotel Roc presidente en cuanto a la situación ambiental y corroborar el nivel de conocimiento de los empleados sobre la temática medioambiental. 
De la revisión teórica realizada cabe destacar lo referido a los indicadores y en específico a los indicadores ambientales. El indicador ambiental es definido como "una expresión específica que aporta información sobre la actuación ambiental de la empresa y sobre sus esfuerzos por influir en tal actuación" (Agencia Europea de Medio Ambiente, 1999). Este tipo de indicador juega un papel básico en la etapa de medición de todo sistema de contabilidad de empresa porque permiten transformar información dispersa y heterogénea en datos útiles, comparables y analizables.

Los indicadores ambientales compensan los extensivos datos ambientales en información crítica que permite monitorear, establecer objetivos, seguir las mejoras de desempeño y realizar comparaciones de diferentes informes. Además, brindan al gerente ambiental y a la alta gerencia, la información requerida para una gran variedad de datos ambientales. Ellos permiten a quienes toman decisiones tener una rápida visión del progreso y de los problemas de protección ambiental que todavía deben ser resueltos. Sobre estas bases, objetivos realistas de mejora de desempeño ambiental pueden ser identificados y cuantificados, lo cual es necesario para controlar los logros actuales. Esta vinculación con el sistema de control tradicional permite el monitoreo de los riesgos ambientales y el desempeño de detección de oportunidades rentables de mejora (Jash, 2002).

Para definir la clasificación de los indicadores ambientales se han usado los criterios de clasificación general de los que parte la Agencia Europea de Medio Ambiente, y que establece una interrelación entre tres conceptos: producción, dirección y medio ambiente. Las decisiones de la dirección de la empresa afectan el desarrollo de las actividades productivas o "de operaciones" de la empresa. Diferentes aspectos de estas actividades productivas tienen posibilidad de afectar el medio ambiente. Estas actividades acaban teniendo impactos concretos y mensurables en el medio ambiente. Tales impactos mesurables deben tenerse en cuenta, a su vez, para reorientar o modificar las decisiones directivas.

Según corresponde a las áreas mencionadas, los indicadores ambientales se dividen en tres grupos: indicadores ambientales de actuación directiva (IAD), indicadores ambientales de actividad productiva (IAP), y los indicadores de condiciones ambientales (ICA). Para elegir los indicadores más adecuados es necesario tener en cuenta una serie de criterios y principios generales. Una segunda forma de describir los indicadores ambientales de empresa es apelar a sus características internas, es decir, definir la tipología a partir de la forma con que tratan la información y presentan los datos. Teniendo en cuenta este criterio, los indicadores pueden expresar sus datos en varios tipos de medidas, en la presente investigación se emplearán solamente términos absolutos y relativos. Medida en términos absolutos: se tratan de indicadores que miden datos básicos en términos absolutos, por ejemplo, consumo de energía en kw, volumen de producción anual, número de miembros de la plantilla, entre otros. Medida en términos relativos: miden la actuación ambiental de la empresa relacionando dos fuentes de información. Para establecer los indicadores relativos debe hacerse una distinción entre dos tipos de indicadores: "cuotas" (también llamadas "proporciones") y "ratios". Las "cuotas" se usan para determinar la proporción del total que corresponde a un subgrupo, mientras que los 
"ratios", por el contrario, relacionan los indicadores absolutos con las unidades operativas que los han causado, por ejemplo, para calcular en una empresa productiva la electricidad (kw) consumida por kilogramo de producto.

Asumiendo los criterios de clasificación aportados por la Agencia Europea de Medio Ambiente, la presente investigación se centrará en los indicadores ambientales de actividad productiva (IAP) o indicadores de comportamiento medioambiental (ICM), y en los indicadores ambientales de actuación directiva (IAD), también conocidos como indicadores de gestión medioambiental (IGM). Los primeros son entendidos como "aquellos que aportan información acerca de la actuación ambiental de las operaciones o de la producción de las empresas; desde los inputs utilizados (materiales, energía, y servicios) hasta los outputs resultantes (productos, servicios, residuos y emisiones)". Por otra parte, los IGM se definen como "los indicadores que brindan información sobre las políticas, gestión de personal, planificación, prácticas y procedimientos a todos los niveles de la empresa, así como sobre las decisiones y acciones relativas a los aspectos ambientales de la organización".

Para la propuesta de indicadores se ha tomado como referencia el Procedimiento para la Identificación de Indicadores Ambientales establecido por el Ministerio Federal de Medio Ambiente de Alemania (BMUB) y la Sociedad Pública de Gestión Ambiental del Gobierno Vasco (2000). Consta de cinco etapas: análisis de situación/ inventario; establecimiento del sistema de indicadores; recopilación de datos y determinación de indicadores; aplicación de los indicadores; y revisión del sistema de indicadores. Como el alcance de la investigación llega hasta la propuesta de indicadores, se han considerado los aspectos de las tres primeras etapas. La trayectoria investigativa se conformó en 5 etapas como y muestra en la figura 1.

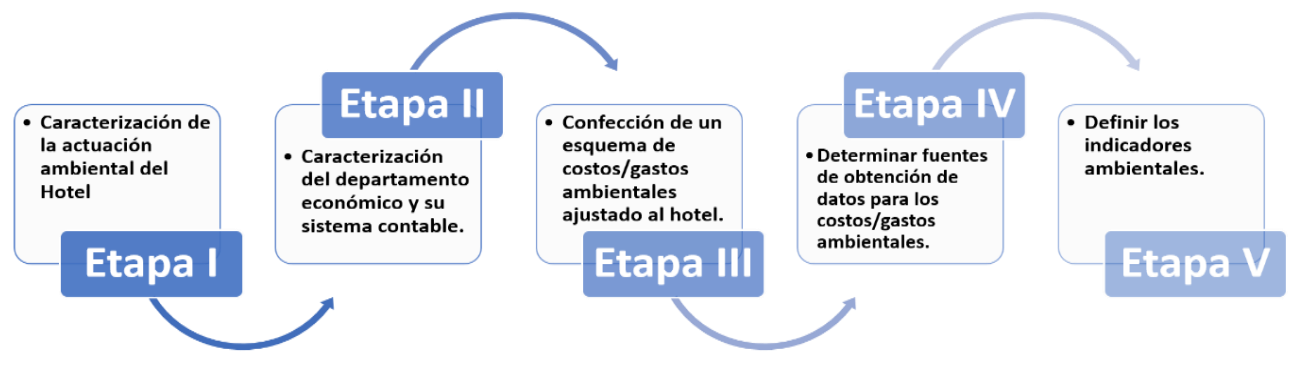

Figura 1: Trayectoria de la investigación. Fuente: Elaboración Propia

\section{Resultados}

Como resultado de las entrevistas realizadas, parte del diagnóstico de la gestión ambiental, el Hotel Roc presidente no aplica un Sistema de Gestión Ambiental y carece de una Comisión de Medio Ambiente. La Especialista de Calidad en conjunto con el Jefe 
de Servicios Técnicos son los que planifican, organizan y controlan las actividades medioambientales que se realizan en el mismo. En estos momentos el hotel no tiene definida una política ambiental y no dispone de un Diagnóstico Ambiental actualizado, por tal motivo se desconocen las no conformidades existentes.

El 25 de mayo de 2010 la Delegación Provincial del CITMA le otorgó al hotel el Certificado de Administración Responsable de la Gestión Ambiental, este no eximía a la entidad de auditorías ambientales y debía ser renovado al término de 1 año, proceso que no se realizó, por tal motivo en estos momentos el hotel no cuenta con esta certificación. El 29 de julio de 2008 en el hotel fue realizada la inspección ambiental estatal, la cual certificó que cumplía con las normativas jurídicas y técnicas con respecto a la protección del medio ambiente, razón por la cual le fue otorgado el Aval Ambiental por parte de la Delegación Provincial del CITMA de la entonces Ciudad de La Habana. Este aval se les reotorga el 21 de marzo de 2018 y hasta la actualidad la instalación no ha sido sancionada o multada en Inspecciones Ambientales Estatales.

Según testimonio del personal del departamento económico del hotel, en la contabilidad no se reflejan elementos de gastos ambientales ni se analizan indicadores ambientales. Solo refieren que se examinan los económicos y de eficiencia, los cuales en el 2017 mostraron resultados satisfactorios de manera general.

\section{Propuesta de esquema de costos/gastos ambientales.}

Se propone un Esquema de Costos Ambientales (Figura 2) que permitirá visualizar el estado completo de los costos ambientales anuales del hotel. Se sugiere que quienes trabajen con este instrumento sean la Especialista de Calidad, algunos trabajadores del departamento económico, el equipo directivo del hotel y el Especialista en Ahorro y Uso Racional de la Energía.

Primeramente, usarán el esquema de forma separada: los contadores identificarán los gastos ambientales para cada cuenta o categoría de cuentas, mientras que el resto de los trabajadores calcularán los costos para cada ambiental (agua, energía, desechos sólidos, etc.).

Posteriormente los resultados serán entrecruzados y revelarán todos los costos del período.

En el esquema se sugiere que los costos identificados sean categorizados por los siguientes medios ambientales: protección del aire ambiental; gestión de portadores energéticos (agua, electricidad, combustible, y gas); gestión de desechos (sólidos y peligrosos); disminución de emisiones (Ruidos); salud y seguridad.

Estos medios ambientales se ajustan a las características del hotel objeto de estudio. Para implementar este esquema en otras entidades, se pueden adicionar otros medios ambientales, por lo que se ajustaría el mismo a la realidad objetiva de cada institución; por ejemplo, para un hotel ubicado en primera línea de playa, sería imprescindible incluir un medio ambiental titulado Protección de la Biodiversidad y el Paisaje. 


\begin{tabular}{|c|c|c|c|c|c|c|c|c|c|c|c|}
\hline $\begin{array}{l}\text { Medios Ambientales } \\
\text { Categoría de costos/gastos } \\
\text { ambientales }\end{array}$ & 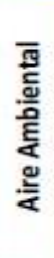 & 总 & 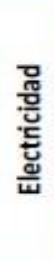 & 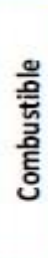 & 号 & 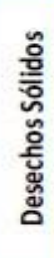 & 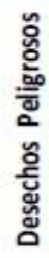 & 을 & 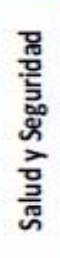 & 气ั & ळ \\
\hline $\begin{array}{l}\text { 1. Tratamiento de medios } \\
\text { ambientales }\end{array}$ & & & & & & & & & & & \\
\hline $\begin{array}{l}\text { 2. Prevención y Gestión } \\
\text { Ambiental }\end{array}$ & & & & & & & & & & & \\
\hline $\begin{array}{l}\text { 3. Costos de los no- } \\
\text { productos }\end{array}$ & & & & & & & & & & & \\
\hline$\Sigma$ Gastos ambientales & & & & & & & & & & & \\
\hline 4. Ingresos ambientales & & & & & & & & & & & \\
\hline$\sum$ Total de ingresos & & & & & & & & & & & \\
\hline
\end{tabular}

Figura 2: Esquema de Costos Ambientales Fuente: Elaboración Propia

Las categorías de costos/gastos ambientales incluidas en el esquema se explica por cada una las partidas o subcuentas que pudieran facilitar la obtención de los valores a otorgarles a los mismos (Figura 3).

Del esquema de costos ambientales diseñado, la participación de costos por medio ambiental y por subcategoría de costos puede ser calculada, por lo tanto, a partir de la aplicación de este esquema se obtendrán diferentes indicadores de costos ambientales que les permitirán a los directivos basarse en información económica para la toma de decisiones en materia ambiental. La fuente de información para ello, en un análisis de los estados financieros y la entrevista realizada se muestra en la figura 4.

Otra de las ventajas de los indicadores de costos ambientales es que, al traducir los asuntos ambientales a costos e ingresos, permitirán expresar variables diferentes en una misma unidad. Por ejemplo, si se deseara sumar o comparar los datos entre los diferentes portadores energéticos sería necesario utilizar factores de conversión para lograr expresar el consumo de todos los portadores en kilovatios hora ( $\mathrm{kWh}$ ) o megavatios hora (MWh). En este caso, para evitar errores de cálculos durante la conversión y facilitar el trabajo de los gestores, utilizar indicadores de costes ambientales puede ser una alternativa viable, de esta manera podría calcularse qué porciento representa el consumo de agua del consumo total de todos los portadores energéticos. 


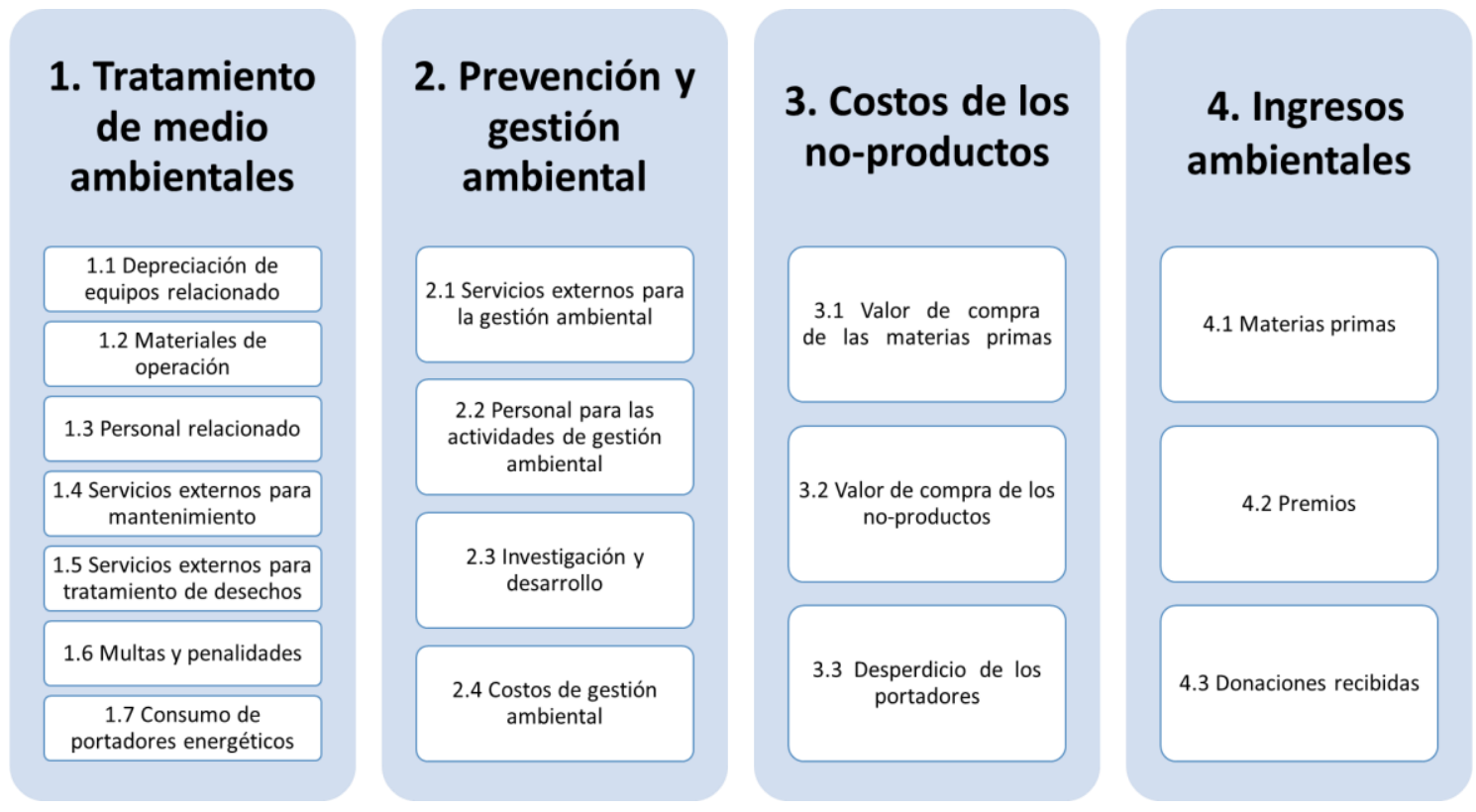

Figura 3: Esquema de costos por categoría

Fuente: Elaboración Propia

\begin{tabular}{|c|c|}
\hline & Irtida o fuent \\
\hline $\begin{array}{l}\text { Aspectos ambientales en el } \\
\text { Balance General. }\end{array}$ & \begin{tabular}{l}
\multicolumn{5}{c}{ Depreciación de los Activos Fijos Tangibles } \\
(subcategoría 1.1 del esquema de costos \\
ambientales).
\end{tabular} \\
\hline $\begin{array}{l}\text { Gastos derivados del } \\
\text { Estado de Resultados. }\end{array}$ & $\begin{array}{l}\text { Gastos Generales y de Administración } \\
\text { (Materiales para Mantenimiento, Servicios } \\
\text { Contratados para Mantenimiento, Otros Servicios } \\
\text { Contratados, Agua y Alcantarillado, Combustible, } \\
\text { Electricidad, y Gas). } \\
\text { Otros Gastos (Gastos Funcionales, Otros } \\
\text { Servicios Contratados, Capacitación y Adiestramiento, } \\
\text { pérdidas de los productos, multas). }\end{array}$ \\
\hline $\begin{array}{l}\text { Gastos derivados de otros } \\
\text { registros del hotel. }\end{array}$ & $\begin{array}{l}\text { Horas de trabajo que invierten cada uno de los } \\
\text { empleados en realizar actividades de mantenimiento de } \\
\text { los equipos ambientales, de tratamiento de los } \\
\text { desechos, y otras acciones dentro de la gestión } \\
\text { ambiental }\end{array}$ \\
\hline
\end{tabular}


Los ingresos por la venta de materia prima $y$ los desechos peligrosos son registrados como Otros Ingresos.

Contratos con la Empresa de Recuperación de Materia Prima.

Registros del departamento de Servicios Técnicos

Figura 4: Fuentes de obtención de datos

Fuente: Elaboración Propia

\section{Propuesta de Indicadores Ambientales.}

La propuesta de indicadores ambientales para el Hotel Roc presidente se deriva de la clasificación general de los indicadores ambientales. En este contexto se han diseñado Indicadores de Comportamiento Ambiental (Figura 5 y 6) e Indicadores de Gestión Ambiental (Figura 7). Para cada indicador se especificó el nombre, la unidad de medida en que debe ser expresado, y las observaciones (fuentes de datos, fórmulas, y otras aclaraciones) que se consideraron pertinentes para una adecuada aplicación de los mismos. El Subdirector General, los jefes de cada departamento implicado, y la Especialista de Calidad serán los responsables de recopilar los datos.

Las unidades de medida de los indicadores están expresadas en términos absolutos y relativos. En el caso de los indicadores ambientales absolutos, estos podrán obtenerse a partir de fuentes de datos existentes en la empresa como, por ejemplo: los registros del departamento de Compras, los consumos de portadores energéticos, la documentación relacionada con el control de la materia prima, el inventario de las especies de plantas, el Plan de Capacitación y Desarrollo, y los registros contables (a partir de la implementación del esquema de costos/gastos ambientales), entre otros.

\begin{tabular}{|c|c|c|c|}
\hline \multicolumn{4}{|c|}{ Indicadores de Comportamiento Medioambiental (Indicadores de entrada) } \\
\hline Actividades & Indicador & Unidades & Observaciones \\
\hline \multicolumn{4}{|c|}{ 1. Uso de productos biodegradables y sustancias peligrosas } \\
\hline \multirow{4}{*}{$\begin{array}{l}1.1 \text { Uso de productos } \\
\text { biodegradables para la } \\
\text { limpieza }\end{array}$} & $\begin{array}{l}\text { Cantidad de productos biodegradables } \\
\text { usados para la limpieza }\end{array}$ & Absoluto (número) & $\begin{array}{l}\text { 1. La información necesaria para los indicadores } \\
\text { propuestos podrá ser encontrada en los registros } \\
\text { del departamento de Compras. }\end{array}$ \\
\hline & $\begin{array}{l}\text { Proporción de productos biodegradables } \\
\text { con respecto al total de productos usados } \\
\text { para la limpieza }\end{array}$ & Relativo (\%) & $\begin{array}{l}\text { 2. Fórmula: (Cantidad de productos biodegradables } \\
\text { para la limpieza/Total de productos para la limpieza) } \\
\text { x } 100\end{array}$ \\
\hline & Consumo de productos biodegradables & Absoluto (kg) & $\begin{array}{l}\text { 3. Deberá ser calculado para cada producto } \\
\text { biodegradable. }\end{array}$ \\
\hline & $\begin{array}{l}\text { Gastos en productos biodegradables usados } \\
\text { para la limpieza }\end{array}$ & $\begin{array}{l}\text { Absoluto (Moneda } \\
\text { total) }\end{array}$ & $\begin{array}{l}\text { 4. Recurrir a la información que aporta el esquema } \\
\text { de costos/gastos ambientales diseñado (1.2). }\end{array}$ \\
\hline
\end{tabular}

Figura 5: Imagen parcial de la tabla propuesta al hotel. Indicadores de entrada. Fuente: Elaboración Propia. 
Desde un punto de vista ecológico, los indicadores absolutos serán el enfoque principal del hotel porque muchos de ellos representarán el consumo de recursos por parte de la empresa y su emisión de sustancias contaminantes, por ejemplo, diversidad de sustancias peligrosas usadas en el hotel, cantidad de agua consumida, gasto por desperdicios de agua, cantidad de desechos peligrosos generados, entre otros. Por otra parte, los indicadores relativos demostrarán si las medidas ambientales dan lugar a mejoras, por ejemplo, en el aumento de las tasas de reciclaje y reutilización de las materias primas, y el ascenso de la proporción de productos biodegradables con respecto al total de productos usados para la limpieza.

Los indicadores de tipo absoluto y relativo permitirán a los directivos del hotel establecer y cuantificar sus objetivos ambientales. La definición de objetivos absolutos podrá ser empleada, por ejemplo, cuando se desee eliminar por completo materiales específicos (sustancias peligrosas) importantes para el medioambiente o cuando se deba hacer un cambio para emplear versiones más seguras para el medio ambiente (productos biodegradables).

\begin{tabular}{|c|c|c|c|}
\hline \multicolumn{4}{|c|}{ Indicadores de Comportamiento Medioambiental (Indicadores de Salidas) } \\
\hline \multicolumn{4}{|c|}{ 3.1 Gestión de desechos } \\
\hline \multirow{8}{*}{$\begin{array}{l}\text { 3.1.1Tratamiento de } \\
\text { desechos sólidos }\end{array}$} & $\begin{array}{l}\text { Cantidad de equipos para el tratamiento de } \\
\text { los desechos }\end{array}$ & Absoluto (número) & $\begin{array}{l}\text { 1.La información para este indicador se puede } \\
\text { encontrar en el departamento de Seguridad y } \\
\text { Protección }\end{array}$ \\
\hline & $\begin{array}{l}\text { Gastos en equipos para el tratamiento de } \\
\text { los desechos }\end{array}$ & $\begin{array}{l}\text { Absoluto (moneda } \\
\text { total) }\end{array}$ & $\begin{array}{l}\text { 2. Acudir a la subcategoría } 1.1 \text { del esquema de } \\
\text { costos ambientales. }\end{array}$ \\
\hline & $\begin{array}{l}\text { Tiempo invertido por los trabajadores de } \\
\text { SSTT y las camareras de área en realizar el } \\
\text { mantenimiento de estos equipos }\end{array}$ & Absoluto (horas) & $\begin{array}{l}\text { 3. El jefe del departamento de SSTT y la Jefa de Ama } \\
\text { de Llaves serán los encargados de determinar esta } \\
\text { información. }\end{array}$ \\
\hline & $\begin{array}{l}\text { Gasto según el tiempo invertido por los } \\
\text { trabajadores de SSTT y camareras de área } \\
\text { en actividades de tratamiento de desechos }\end{array}$ & $\begin{array}{l}\text { Absoluto (moneda } \\
\text { total) }\end{array}$ & $\begin{array}{l}\text { 4. La subcategoría } 1.1 \text { contiene los datos necesarios } \\
\text { para este indicador. }\end{array}$ \\
\hline & $\begin{array}{l}\text { Cantidad de empresas contratadas para el } \\
\text { mantenimiento de estos equipos }\end{array}$ & \multirow[t]{2}{*}{ Absoluto (número) } & \multirow{2}{*}{$\begin{array}{l}\text { 5. El jefe de Seguridad y Protección y el de Servicios } \\
\text { Técnicos son los que dominan esta información } \\
\text { dentro del hotel. }\end{array}$} \\
\hline & $\begin{array}{l}\text { Cantidad de empresas contratadas para el } \\
\text { tratamiento de desechos }\end{array}$ & & \\
\hline & $\begin{array}{l}\text { Gastos en servicios externos para el } \\
\text { mantenimiento de estos equipos }\end{array}$ & \multirow[t]{2}{*}{$\begin{array}{l}\text { Absoluto (moneda } \\
\text { total) }\end{array}$} & \multirow{2}{*}{$\begin{array}{l}\text { 6. En el esquema de costos/gastos ambientales las } \\
\text { subcategorías } 1.4 \text { y } 1.5 \text { son las que contienen los } \\
\text { datos de estos indicadores. }\end{array}$} \\
\hline & $\begin{array}{l}\text { Gastos en servicios externos para } \\
\text { tratamiento de desechos }\end{array}$ & & \\
\hline $\begin{array}{l}\text { 3.1.2 Aprovechamiento de } \\
\text { desechos inorgánicos para }\end{array}$ & $\begin{array}{l}\text { Cantidad total de desechos inorgánicos } \\
\text { generados }\end{array}$ & Absoluto (kg) & $\begin{array}{l}\text { 1. La información necesaria para el cálculo de estos } \\
\text { indicadores se encuentra en el departamento de }\end{array}$ \\
\hline
\end{tabular}

Figura 6: Imagen parcial de la tabla propuesta al hotel. Indicadores de salida. Fuente: Elaboración Propia.

Las cifras de objetivos relativos serán idóneas cuando un consumo específico no se pueda evitar completamente, pero en proporción con el volumen de operación pueda emplearse más eficientemente o utilizarse en menor porcentaje, por ejemplo, para el consumo de agua, energía o gas por habitaciones ocupadas. 


\begin{tabular}{|c|c|c|c|}
\hline \multicolumn{4}{|c|}{ Indicadores de Gestión Medioambiental } \\
\hline Actividades & Indicador & Unidades & Observaciones \\
\hline \multirow{6}{*}{$\begin{array}{l}\text { 1. Realización de } \\
\text { auditorías ambientales }\end{array}$} & $\begin{array}{l}\text { Cantidad de auditorías ambientales } \\
\text { efectuadas }\end{array}$ & \multirow{4}{*}{ Absoluto (número) } & \multirow{4}{*}{$\begin{array}{l}\text { 1. Recientemente el hotel fue inspeccionado por el } \\
\text { CITMA para verificar el cumplimiento de los } \\
\text { requisitos que deben cumplirse para la obtención } \\
\text { del Aval Ambiental. Dicha inspección pudiera ser } \\
\text { tomada en cuenta para los indicadores propuestos } \\
\text { en este bloque. }\end{array}$} \\
\hline & $\begin{array}{l}\text { Desviaciones descubiertas en auditorías } \\
\text { ambientales }\end{array}$ & & \\
\hline & $\begin{array}{l}\text { Medidas de mejora propuestas para } \\
\text { cuestiones ambientales }\end{array}$ & & \\
\hline & Medidas correctivas llevadas a cabo & & \\
\hline & $\begin{array}{l}\text { Proporción de propuestas de mejoras } \\
\text { llevadas a cabo }\end{array}$ & \multirow[t]{2}{*}{ Relativo (\%) } & $\begin{array}{l}\text { 2. Fórmula: (Número de medidas correctivas } \\
\text { llevadas a cabo / Total de medidas de mejora } \\
\text { propuestas ) × } 100\end{array}$ \\
\hline & Grado de consecución general de los & & 3. Fórmula: (Número de objetivos ambientales \\
\hline
\end{tabular}

Figura 7: Imagen parcial de la tabla propuesta al hotel. Indicadores de Gestión ambiental.

Fuente: Elaboración Propia.

\section{Consideraciones generales.}

La selección de los indicadores propuestos ha sido una selección inicial basada en datos existentes en el hotel (fuente de información interna). Los mismos podrán irse perfeccionando con el cursar del tiempo hasta conformar un complejo sistema de indicadores que brinde información sobre el comportamiento ambiental de la empresa. Podrán calcularse con una frecuencia anual para hacerlos comparables años tras año. Solo desarrollándolos de forma periódica podrán usarse como instrumento de gestión eficaz.

Las comparaciones de series temporales permitirán detectar puntos débiles y poner de manifiesto potenciales de optimización, lo que asegura que se fijen objetivos realistas.

La medición de los aspectos ambientales del hotel a través de los indicadores permitirá determinar objetivos cuantificables. El examen periódico de los indicadores ambientales no solo apoyará el seguimiento y control sistemático de los objetivos, sino que también proporcionará declaraciones sobre la mejora continua de cuestiones ambientales importantes. Los indicadores facilitarán la elaboración de informes y declaraciones ambientales, ya que permiten representar hechos de manera compacta y concisa.

\section{Conclusiones}

- Las herramientas elaboradas devienen en instrumento para el comienzo de la cuantificación de los costos ambientales lo que permitirá la toma de decisiones y la mejora implementación de un sistema de Gestión ambiental. Los indicadores de costos ambientales propuestos contribuirán a traducir los asuntos ambientales del hotel en costos, lo que permitirá aunar los objetivos ambientales que se tracen, con la rentabilidad económica del hotel. El cálculo de los costos de las actividades ambientales en conjunto con la aplicación de indicadores de comportamiento y de gestión ambiental (absolutos y relativos) permitirá la formulación de políticas para 
la toma de decisiones que contribuyan a un mejor desempeño ambiental del hotel. El esquema de costos/gastos ambientales propuesto permitirá identificar los gastos que generan las actividades empresariales para el cuidado y conservación del medio ambiente, a partir de su separación de los elementos de gastos empresariales. La contabilidad del Hotel Roc presidente no tiene en cuenta los principios de la contabilidad ambiental, por lo que el cálculo de los costos ambientales constituye una propuesta novedosa para la entidad.

\section{Referencias bibliográficas}

Agencia Europea de Medio Ambiente. (1999). Contabilidad Ambiental: Medida, evaluación y comunicación de la actuación ambiental de la empresa. Barcelona, España.

Cañizares, M. (2015). Tesis en opción al grado científico de Doctor en Ciencias Contables y Finanzas. Procedimiento para cuantificar los costos de las actividades ambientales en la gestión de sostenibilidad del recurso agua potable. . La Habana, Cuba.

CITMA. (1997). Gaceta Oficial de la República de Cuba. Ley No. 81 del Medio Ambiente (7), Edición Extraordinaria. La Habana.

Feria, Y. (2012). Trabajo de diploma en opción al título de Licenciado en Turismo. Evaluación ambiental en la Sucursal Emprestur S.A. de aseguramiento. La Habana, Cuba.

Hernández, R. (2006). Metodología de la investigación. La Habana: Editorial Ciencias Médicas.

IFAC. (2005). International Financial Accounting Comitee. www.ifac.org

IHOBE-BMUB. (2000). Guía de indicadores medioambientales para la empresa.

https://alojamientos.uva.es/guia_docente/uploads/2013/430/52300/

Jash, C. (2002). Environmental Management Accounting. (G. M. Scavone, Trad.) Alemania.

LLul, A. (2001). Tesis Doctoral. Contabilidad Medioambiental y Desarrollo Sostenible en el Sector Turístico. Islas Baleares.

Martín, R. (2010). Principios, Organización y Práctica del Turismo. La Habana, Cuba

Noa, M., \& Salas, F. (2010). La contabilidad ambiental en Cuba, perspectivas y desafíos. Desarrollo local sostenible, 5. Cuba.

Organización Internacional de Normalización. (2015). Norma Internacional ISO 14001. Sistema de gestión ambiental- Requisitos con orientación para su uso, 3. Ginebra, Suiza. 
Paula, L. (2011). Trabajo de diploma. Factores de Riesgos Medioambientales de la actividad turística en el destino "Playa del Este" y su relación con la contabilidad medioambiental. La Habana, Cuba.

Pelegrín, A., \& Acosta. (2011). Revista Confin Habana. Recuperado el 28 de febrero de 2018, de La vivienda biohabitable, un enfoque medioambiental a partir del presupuesto: http://www.cofinhab.uh.cu/index.php/cofin/article/view/23

Perelló, J. (2005). Introducción a la Investigación de Mercados Turísticos. La Habana, Cuba.

Pérez, Y. (2013). Trabajo de diploma en opción al título de Licenciado en Turismo. Vulnerabilidad de las instalaciones turísticas del Malecón Habanero ante los peligros naturales que afectan el área. La Habana, Cuba.

Suárez, D. (2013). Trabajo de diploma en opción al título de Licenciado en Turismo. Vulnerabilidad de las instalaciones turísticas del litoral de Miramar ante los peligros naturales que afectan el área. La Habana, Cuba.

Vargas, T. (2013). Tesis presentada en opción al grado de Doctor en Ciencias Económicas. Modelo de Gestión de la capacitación orientado al desarrollo de competencias para el turismo sostenible en el ámbito local, con enfoque de organización que aprende. Pinar del Río, Cuba.

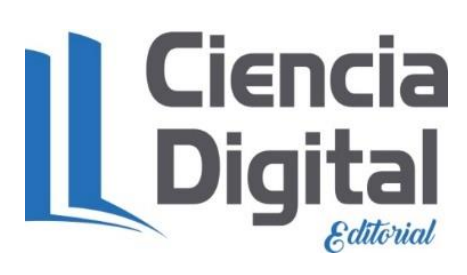




\section{PARA CITAR EL ARTÍCULO INDEXADO.}

Perera Conde, L., Nogueiras Valdés, A., \& Alcober Álvarez, R. R. (2021). Indicadores para la medición de los costos ambientales en entidades de alojamiento turístico: Una necesidad ante la sostenibilidad del uso de los recursos. Explorador Digital, 5(1), 185200. https://doi.org/10.33262/exploradordigital.v5i1.1497

\section{\Cigncia}

El artículo que se publica es de exclusiva responsabilidad de los autores y no necesariamente reflejan el pensamiento de la Revista Explorador Digital.

El artículo queda en propiedad de la revista y, por tanto, su publicación parcial y/o total en otro medio tiene que ser autorizado por el director de la Revista Explorador Digital.
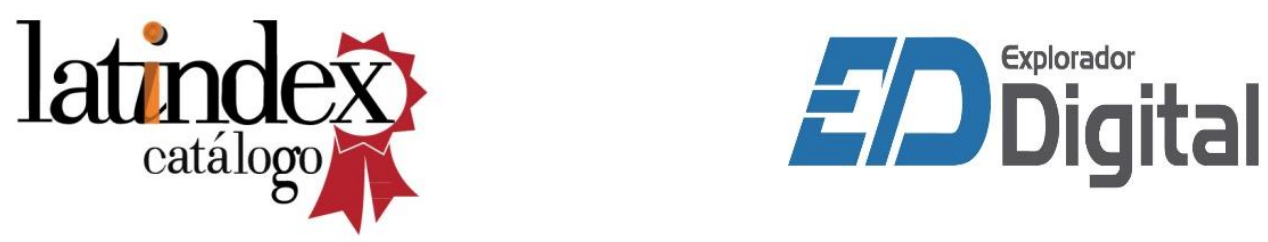